\section{SP0122 AUTOIMMUNE PHENOMENA ASSOCIATED WITH BIOLOGICAL AGENTS}

P. Quartier ${ }^{1,2} .{ }^{1}$ Pediatric Immunology-Hematology and Rheumatology Unit, Necker-Enfants Malades Hospital; ${ }^{2}$ IMAGINE Institute, Paris-Descartes University, Paris, France

Biologic agents are being increasingly used in pediatric rheumatology, particularly TNF antagonists but also abatacept, tocilizumab, interleukin (IL)-1 antagonists and some other drugs. In Juvenile Idiopathic Arthritis (JIA) and some autoinflammatory diseases, data from phase 3 and extension trials or from cohorts such as Pharmachild allow to prospectively collect information on adverse events "of special interest", including autoimmune complications. A few patients develop autoimmune/dysimmune features while on biologics, as seen in adults, including central nervous system lesions, inflammatory bowel disease or psoriasis. In addition, in patients with systemic-onset $\mathrm{JIA}$, anti- IL-1 treatment is usually associated with the appearance of a type 1 interferon signature (gene expression analyses) which might in some cases favour lupus-like autoimmune features. On the other hand, among patients with early-onset arthritis, vasculitis, recurrent fever or other inflammatory manifestations, an increased number of children are diagnosed with complex monogenic diseases resulting in auto-inflammation, immune deficiency and autoimmunity. In such cases, biologics might not be responsible for the occurrence of autoimmune features that may sometimes be diagnosed on treatment. This distinction is important as biologics are useful treatments also in some of these patients, as was shown in patients with a diagnosis of Systemic-onset JIA and ANCA-associated glomerulonephritis in whom anti-IL-1 treatment was beneficial. It was also more recently shown in patients with lipopolysaccharide-responsive beige-like anchor (LRBA) mutations associated with autoimmunity and inflammation, including polyarthritis: as LRBA is a partner of cytotoxic-T lymphocyte antigen-4 (CTLA4),. abatacept has been used as a targeted treatment and shown efficacy.

We hence aim to discuss the way to explore patients who develop autoimmune features while on biologics in order to take the right decisions regarding treatment maintenance, withdrawal or modification and regarding patients follow-up.

Disclosure of Interest: P. Quartier Grant/research support from: Abbvie, Novartis, Pfizer, Roche, Consultant for: Abbvie, Novartis, Sanofi, SOBI, Speakers bureau: Abbvie, BMS, Novartis, PfiZer, Roche, SOBI DOI: 10.1136/annrheumdis-2017-eular.7231

\section{FRIDAY, 16 JUNE 2017 \\ Health equity and economy - a vital relationship}

\section{SP0123 UNCOVERING THE EQUITY GAP IN RHEUMATIC AND MUSCULOSKELETAL DISEASES}

P. Putrik. Rheumatology, Maastricht University Medical Center (MUMC), Maastricht, Netherlands

The aim of this lecture is to discuss the current evidence on the socio-economic inequities in disease outcomes in RMDs. Socio-economic determinants at the individual and country level will be considered, as well as the interplay between these factors. In particular, attention will be given to the role of different socio-economic factors in the access to biologic DMARDs in rheumatoid arthritis. Disclosure of Interest: None declared

DOI: 10.1136/annrheumdis-2017-eular.7164

\section{SP0124 HEALTH ECONOMICS AND HEALTH EQUITY: TWO COMPLEMENTARY DISCIPLINES}

B. Fautrel ${ }^{1,2,3} \cdot{ }^{1}$ Rheumatology, APHP - CHU Pitié Salpétrière; ${ }^{2}$ Rheumatology, UPMC - Pierre and Marie Curie University; ${ }^{3}$ CRI-IMIDIATE Clinical Research Network, FCRIN Clinical Research Infrastructure Networks, Paris, France

Health equity in the one hand refers to the absence of systematic disparities in health between different social groups in a given Society, a province, a country or a group of country. Health inequity thus corresponds to a situation in which health services are not similarly available to all people with the same health conditions and health needs, due to individual personal or socioeconomic characteristics. Health economics in the other hand focuses on how to allocate health budgets in order to maximize the general health of the population as a whole. With regards to this, no specific attention is dedicated to socially disadvantaged subgroups. In addition, the most visible action in the field of health economics was the valorization of therapeutic innovation, i.e., the determination of its price not on production costs but on the value associated with this innovation.

Economic evaluation - i.e., determination of incremental cost-effectiveness ration - has lead during the last 20 years to substantial financial pressure on health care systems with dramatic increase in health expenditures mainly due to the costs of therapeutic innovation. Several studies have shown that such a process may increase health inequities within a country or a group of countries if specific actions are not taken to maintain or improve treatment availability and access to care to all the population members whatever their social, educational and economic characteristics.

Disclosure of Interest: None declared
DOI: 10.1136/annrheumdis-2017-eular.7280

\section{FRIDAY, 16 JUNE 2017 \\ Biomarkers in cardiovascular rheumatology - state-of-the-art 2017}

\section{SP0125 INFLAMMATION AND CARDIOVASCULAR DISEASE - RELEVANT METABOLIC BIOMARKERS}

\section{E. Choy. Section of Rheumatology, Cardiff University, Cardiff, United Kingdom}

Patients with RA have increased mortality compared with the general population mostly due to higher cardiovascular disease (CVD), which is up to $50 \%$ more frequent [1]. Even after adjusting for traditional cardiovascular risk factors such as smoking, diabetes and hypertension, the risk for CVD is increased by up to twofold compared with the normal population [2]. Whilst traditional cardiovascular risk factors, contribute to the increased risk of mortality in RA patients, they do not fully explain increase in cardiovascular risk $[3,4]$. European League Against Rheumatism (EULAR) recommend regular assessment of cardiovascular risk in patients with RA [5]. Since traditional cardiovascular risk factor assessment equations, such as Framingham and the Systematic Coronary Risk Evaluation Score (SCORE) models, underestimate cardiovascular risk in RA, EULAR recommends multiplying such traditional cardiovascular risk scores by 1.5 for patients with RA. Such adjustment operates at the population level. Ideally, cardiovascular biomarkers that can predict future cardiovascular event in the individual patient will improve screening and management.

Biomarkers of cardiovascular disease can be divided into five major categories: lipids, inflammation, endocrine, vascular and prothrombotic [7]. HDL and LDL are used in routine clinical practice. However, they do not predict future cardiovascular events in patients with RA as the levels of $\mathrm{HDL}$ and $\mathrm{LDL}$ are suppressed during inflammation [8]. The ratio of HDL/LDL or total cholesterol/HDL is less affected by inflammation. Other lipid biomarkers include apolipoprotein A-1, apolipoprotein B, cholesterol ester transfer protein lipoprotein-associated phospholipase A2, small-dense LDL and paraxonase-1. They have been measured in patients with RA but their precise value in predicting cardiovascular risk in RA has not been determined.

High level of inflammation as measured by ESR and CRP is associated with increased cardiovascular risk in patients with RA. EULAR recommended adequate suppression of inflammation as a key strategy to reduce cardiovascular events [5]. Disease flares increased cumulative cardiovascular risk [9]. Many inflammatory mediators are elevated in RA, whether they can add to traditional cardiovascular risk score to improve individual risk prediction should be evaluated.

The vascular biomarker of cardiovascular disease, VCAM-1, has also been shown to elevated in patients with RA. High level of VCAM-1 was associated with high cardiovascular risk score[ix].

Metabolic syndrome is common in patients with inflammatory arthritis. Insulin resistance is a feature of metabolic syndrome. Fibrinogen and other prothrombotic molecules are part of the acute phase response, their levels are elevated in RA. Neither endocrine nor prothrombotic factors have been studied systematically in RA.

References:

[1] Meune C, Touze E, Trinquart L, Allanore Y. Rheumatology 2009 Oct; 48(10):1309-13.

[2] Gabriel SE. The American Journal of Medicine 2008 Oct;121(10 Suppl 1):S9-14.

[3] Dessein PH, Joffe BI, Veller MG, Stevens BA, Tobias M, Reddi K, et al. The Journal of rheumatology $2005 \mathrm{Mar} ; 32(3): 435-42$.

[4] del Rincon ID, Williams K, Stern MP, et al. Arthritis Rheum. 2001;44:27372745.

[5] Agca R, Heslinga SC, Rollefstad S et al. Ann Rheum Dis. 2017 Jan;76(1):1728.

[6] Montgomery JE and Brown JR. Vascular Health and Risk Management. 2013:9 37-45

[7] Choy E and Sattar N. Ann Rheum Dis. 2009 Apr;68(4):460-9.

[8] Myasoedova E, et al. Ann Rheum Dis. 2016 Mar;75(3):560-5.

[9] Davies RD et al ACR 2016 abstract no 1447.

Disclosure of Interest: E. Choy Grant/research support from: Roche, UCB, Pfizer, Biocancer, Consultant for: Amgen, Biogen, BMS, Boehringer Ingelheim, Celgene, Chugai Pharma, Eli Lilly, Hospita, I Janssen, Napp, Novimmune, Novartis, Pfizer, Regeneron, Roche, R-Pharm, Sanofi-Aventis, Tonix and UCB., Speakers bureau: Amgen, BMS, Boehringer Ingelheim, Chugai Pharma, Eli Lilly, Hospira, MSD, Novartis, Pfizer, Regeneron, Roche, Sanofi-Aventis, and UCB. DOI: 10.1136/annrheumdis-2017-eular.7237

\section{SP0126 THE VESSEL WALL IN IMIDS - NEW EMERGING VASCULAR MARKERS}

Z. Szekanecz. Department of Rheumatology, University of Debrecen, Faculty of Medicine, Debrecen, Hungary

Cardiovascular disease dependent on inflammatory accelerated atherosclerosis 